Hospital Practice

\title{
Estimation of glomerular filtration rate from the serum creatinine concentration
}

\author{
Stephen A. Smith \\ Department of Medicine, Flinders Medical Centre, Bedford Park, South Australia
}

\begin{abstract}
Summary: In patients with renal failure the dose of renally excreted drugs should be reduced in proportion with the degree of impairment of glomerular filtration: in this situation it is appropriate to measure the glomerular filtration rate in order to determine accurately the correct dose. However, as 24 hour urine based creatinine clearance measurements are time consuming and inaccurate, the parameter most often used to estimate renal function in clinical practice is the serum creatinine concentration. An isolated serum creatinine concentration is unsatisfactory for this purpose as it depends on creatinine production, which is related to muscle mass, as well as on renal elimination of creatinine.

A variety of formulae and nomograms have been devised to assist clinicians in predicting the glomerular filtration rate from a stable serum creatinine concentration and the patient's sex, age and weight (or height). Although some of these formulae have been available for over 15 years, the majority of doctors cannot estimate accurately the glomerular filtration rate from these parameters.

It is proposed in this paper that biochemistry results computers should employ one of these formulae to calculate the glomerular filtration rate $/ 70 \mathrm{~kg}$, using the age and sex information provided on the request form, each time a serum creatinine concentration is reported. These formulae are invalid in several well defined clinical situations which could be briefly outlined on the report.
\end{abstract}

\section{Introduction}

An accurate estimate of renal function is a vital tool in the management of patients with renal disease or when prescribing potentially toxic drugs that are excreted by the kidney. For this purpose it has been traditionally appropriate to measure or to estimate the glomerular filtration rate. Twenty four hour creatinine clearance measurements can be used to estimate glomerular filtration rate but the technique is time consuming, inconvenient and frequently inaccurate. ${ }^{1,2}$

Thus the parameter most often used to estimate renal function in clinical practice is the serum creatinine concentration. Unfortunately serum creatinine concentration, when considered in isolation, is unsatisfactory as it is not directly

Correspondence: S.A. Smith, M.R.C.P.(UK)

Dr S.A. Smith holds a British Heart Foundation/ Australian National Heart Foundation Travelling Fellowship.

Accepted: 20 October 1987 related to the glomerular filtration rate. Serum creatinine concentration depends upon the balance between the production of creatinine and its excretion by the kidneys. Creatinine production is largely determined by muscle mass, which in turn is related to age, sex and weight, and will vary from patient to patient. In addition serum creatinine concentration is related to glomerular filtration rate in a reciprocal fashion; when renal function is normal or only mildly impaired, small changes in serum creatinine concentration represent large changes in glomerular filtration rate. In patients with renal impairment, the dose of renally excreted drugs should be reduced in proportion with the reduction in glomerular filtration: in this situation it is necessary to measure or estimate the glomerular filtration rate in order to determine accurately the appropriate dose.

- Most biochemistry laboratories produce normal ranges for their serum creatinine assay based upon the mean \pm 2 standard deviations of the results obtained from a large, it is hoped, heterogeneous

(C) The Fellowship of Postgraduate Medicine, 1988 
population. This leads to the erroneous belief that all patients with serum creatinine values within the normal range have normal renal function.

Thus clinicians will be misled greatly if they base their clinical assessment of a patient's renal function on the serum creatinine concentration without fully understanding the effects of muscle mass on this parameter.

Can clinicians accurately estimate renal function from the serum creatinine concentration?

The importance of this problem is illustrated by the results of a postal survey that I conducted in a large British teaching hospital. A questionnaire was sent to 128 doctors (both junior and senior), working in clinical, medical and surgical specialties, outlining details of the ages, sex, weights and serum creatinine concentrations of five hypothetical patients (Table I). The doctors were invited to estimate each patient's renal function, in terms of glomerular filtration rate, from these parameters. The correct answers were determined using an empirical formulae described by Hull et $a l^{3}$ as follows:

$$
\begin{gathered}
\mathrm{GFR}_{(\mathrm{m})} / 70 \mathrm{~kg}=88 \times \frac{(145-\mathrm{Age})}{\mathrm{Cr}_{\mathrm{s}}}-3 \\
\mathrm{GFR}_{(\mathrm{f})} / 70 \mathrm{~kg}=0.85\left(\mathrm{GFR}_{(\mathrm{m})} / 70 \mathrm{~kg}\right)
\end{gathered}
$$

where $G R_{(m)}$ was the glomerular filtration rate for men $(\mathrm{ml} / \mathrm{min})$, $G_{F R}$ (f) was the glomerular filtration rate for women $(\mathrm{ml} / \mathrm{min})$ and $\mathrm{Cr}_{\mathrm{s}}$ was the serum creatinine concentration $(\mu \mathrm{mol} / \mathrm{l})$.

Figure 1 shows individual estimates of glomerular filtration rate for each of the five patients. The most striking feature was the wide scatter of answers. The majority of doctors overestimated glomerular filtration rate in patients $1,3,4$ and 5 by failing to account for increased age (patient 5), low weight

Table I Characteristics of the 5 hypothetical subjects included in the questionnaire. Doctors were invited to estimate glomerular filtration rate. The answers are shown in Figure 1.

\begin{tabular}{ccccc}
\hline $\begin{array}{c}\text { Subject } \\
\text { number }\end{array}$ & $\begin{array}{c}\text { Age } \\
(\text { years })\end{array}$ & $\begin{array}{c}\text { Sex } \\
(M / F)\end{array}$ & $\begin{array}{c}\text { Weight } \\
(\mathrm{kg})\end{array}$ & $\begin{array}{c}\text { Serum creatinine } \\
(\mu \mathrm{mol} / \mathrm{l})\end{array}$ \\
\hline 1 & 51 & $\mathrm{~F}$ & 75 & 85 \\
2 & 22 & $\mathrm{M}$ & 95 & 140 \\
3 & 18 & $\mathrm{~F}$ & 60 & 160 \\
4 & 40 & $\mathrm{~F}$ & 55 & 110 \\
5 & 78 & $\mathrm{M}$ & 65 & 170 \\
\hline
\end{tabular}

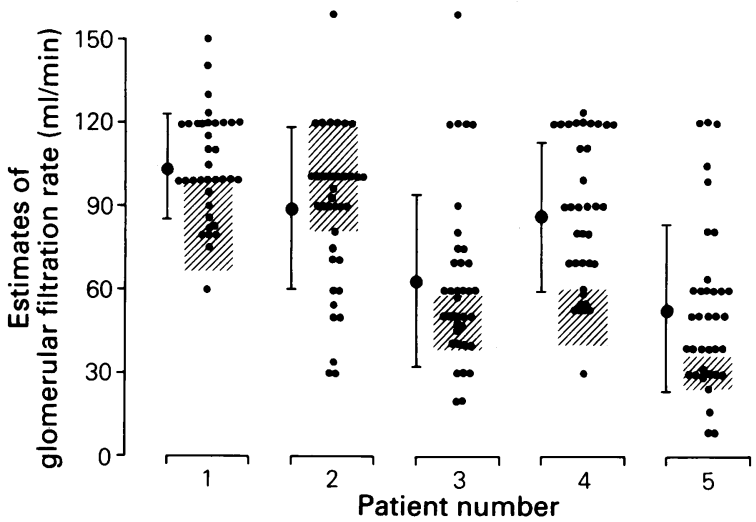

Figure 1 Individual estimates of glomerular filtration rate for each of the 5 patients described in Table I. The correct answers $\pm 20 \%$ are shown by the shaded areas. The vertical bars represent the mean \pm s.d.

(patient 4) and female sex (patients 1, 3 and 4). Most underestimated the glomerular filtration rate in patient 2 for the same reasons.

It appeared that many doctors had considerable difficulty in accurately interpreting the serum creatinine concentration. Whilst some of the estimates of glomerular filtration rate were reasonably close, many more were so inaccurate that prescription of appropriate doses of renally excreted drugs such as digoxin and aminoglycoside antibiotics by these doctors would be more a matter of chance than of judgement.

\section{Using serum creatinine concentration to estimate glomerular filtration rate}

Creatinine clearance, traditionally regarded as the best method for determining glomerular filtration rate in routine clinical practice, is imprecise and unpredictably inaccurate. Errors may arise because of active secretion of creatinine by renal tubules, $\mathbf{4 , 5}$ inaccurate urine collection ${ }^{6,7}$ and fluctuations in serum creatinine throughout the day ${ }^{8,9}$ Creatinine clearance measurements made using routine laboratory services and hospital outpatient facilities have coefficients of variation as high as $27 \%,{ }^{2}$ whereas isolated serum creatinine concentrations have day to day coefficients of variation of approximately $7 \% .^{10}$

The idea of using single serum creatinine concentrations to determine glomerular filtration rate is attractive as many of the problems inherent in the measurement of creatinine clearance are avoided. The main difficulty with using serum creatinine concentrations has been correcting for 
the influence of muscle mass. One solution would be to produce separate series of reference ranges for men and women of different ages and weights. These reference ranges are not yet complete although some work has been published in this area. ${ }^{11-15}$

An alternative approach has been to devise formulae or nomograms that compensate for variations in creatinine production and allow prediction of glomerular filtration rate from a subject's age, sex and weight (or height) and a stable serum creatinine value. Several such formulae have been proposed over the last 15 years. ${ }^{3 \cdot 16-24}$ They have all been derived empirically and then tested prospectively by comparing their results with creatinine clearance or other measures of glomerular filtration rate. The first clinical application of a formula in adults was described by Jelliffe $^{20}$ in 1971. A formula is available for use in children, where sex is a less important determinant of muscle mass, relating height and serum creatinine to glomerular filtration rate. ${ }^{21}$ Over the last decade these formulae have been modified and improved. Most give reasonable results with correlation coefficients of greater than $0.9^{3}$ when compared with other methods such as measurement of creatinine clearance.

The Hull formula, ${ }^{3}$ which was used to determine the answers in my questionnaire, is particularly useful because it is simple and it has been validated by comparison with creatinine clearance over the whole spectrum of renal function, from normal to severe impairment, in patients with wide ranges of age and weight. The results obtained with this formula compare favourably with those from earlier formulae. ${ }^{3}$

Recently there has been vigorous debate about the relative merits of using the serum creatinine concentration or formab creatinine clearance measurements to determine renal function. Strong arguments have been tendered both for ${ }^{2}$ and against $^{25}$ scrapping the 24 hour urine technique. At present it seems reasonable that those who wish to should continue to use the urine collection technique. However it is important that they should also be aware of, and able to apply, the alternative serum creatinine methods.

\section{Problems in the interpretation of serum creatinine concentration}

It is important to realise that serum creatinine concentration cannot be used reliably to calculate glomerular filtration rate in conjunction with the Hull formula (or any of the other formulae or nomograms) in all patients. Errors will arise in several well defined clinical situations:

(1) In acute renal failure ${ }^{26}$ when serum creatinine concentrations are rapidly changing (either $c$ upwards or downwards). Formulae are only $\ddot{\overrightarrow{\vec{*}}}$ valid when a 'steady state' exists. Although $\stackrel{\stackrel{\rho}{口}}{\stackrel{2}{ }}$ more complicated formulae which take account of changing serum creatinine have been devised, ${ }^{27}$ this effectively means that renal function should not have changed greatly over the previous 4 days. The same restrictions also apply to 24 hour urine creatinine clearance.

(2) Within 8 hours of a meat-containing meal Serum creatinine concentration changes throughout the day in subjects taking a meatcontaining diet. Increases of up to $80 \%$ were seen following a test meal containing $300 \mathrm{~g}$ of cooked beef ${ }^{8}$ and $30 \%$ increases have been described following ordinary hospital meals. ${ }^{9}$ These studies were undertaken in subjects with normal renal function: the percentage change would become much smaller as the baseline serum creatinine increases in subjects with renal impairment. If meat is avoided the serum creatinine concentration remains relatively constant. ${ }^{28-30}$ Early morning creatinine concentrations are remarkably constant and are preferred when accurate prediction of renæ function is important. As serum creatinine rise with more severe renal impairment, the significance of dietary meat diminishes.

(3) Strenuous exercise may increase the serum creatinine concentration by $14 \%{ }^{31}$

(4) In severe oedematous states when muscle mass cannot be predicted from body weight. The same may be true for grossly obese subjects and women during the later stages of pregnancy.

(5) In patients with marked muscle wasting. ${ }^{32}$

(6) In children where the influence of sex on muscle mass is less important. Alternative formulae are available for use in children. ${ }^{21}$

(7) In patients with liver disease. ${ }^{3}$ The reasons for inaccuracy of these formulae in liver disease are uncertain although muscle wasting and oedema may play a part. In patients with marked jaundice bilirubin may interfere with the creatinine assay. ${ }^{33}$

(8) In ketotic patients where serum creatinine concentration may be falsely elevated by interference with the creatinine assay. ${ }^{34}$

(9) Drugs such as salicylate, ${ }^{35}$ one or both of the components of co-trimoxazole ${ }^{36}$ and 0 cimetidine $^{37}$ can inhibit tubular secretion of creatinine, increasing plasma creatinine concentration without any change in glomerular function. 


\section{Conclusion}

Several formulae are now available which allow accurate prediction of glomerular filtration rate from a single serum creatinine concentration. These formulae can be applied in the majority of patients although they are invalid in a number of clearly defined clinical situations. It seems that despite the existence of these formulae most hospital doctors are unable to interpret serum creatinine values in terms of glomerular filtration rate.

It is probably unreasonable to expect all doctors to remember a formula and use it each time they receive a serum creatinine result. However, it should be possible for biochemistry computers to use the age and sex information that is provided on most request forms, and to utilize one of the available

\section{References}

1. Brochner Mortensen, I. \& Rodbro, P. Selection of routine methods for determination of glomerular filtration rate in adult patients. Scand J Clin Lab Invest 1976, 36: 35-43.

2. Payne, R.B. Creatinine clearance: a redundant investigation. Ann Clin Biochem 1986, 23: 243-250.

3. Hull, J.H., Hak, L.J., Koch, G.G., Wargin, W.A., Chi, S.L. \& Mattocks, A.M. Influence of range of renal function and liver disease on predictability of creatinine clearance. Clin Pharmacol Ther 1981, 29: 516-521.

4. Wessen, L.G. Physiology of the Human Kidney. Grune and Stratton, New York, 1969, 189-198.

5. Anderson, C.F., Jaekes, D.M., Ballon, H.S. et al. Renal handling of creatinine in nephrotic and nonnephrotic patients. Clin Sci 1970, 38: 555-562.

6. de Wardener, H.E. In The Kidney. Churchill Livingstone, Edinburgh, 1985, p. 40-41.

7. Winterborn, M.H., Beetham, R. \& White, R.H.R. Comparison of plasma disappearance and standard clearance techniques for measuring glomerular filtration rate in children with vesicoureteric reflux. Clin Nephrol 1977, 7: 262-270.

8. Jacobson, F.K., Christensen, C.K., Mogensen, C.E. et al. Pronounced increase in serum creatinine concentration after eating cooked meat. $B r$ Med $J$ 1979, i: 1049-1050.

9. Pasternack, A. \& Kuhlback, B. Diurnal variations of serum and urine creatine and creatinine. Scand J Clin Lab Invest 1971, 27: 1-7.

10. Rosano, T.G. \& Brown, H.H. Analytical and biological variability of serum creatinine and creatinine clearance: implications for clinical interpretation. Clin Chem 1982, 28: 2330-2331.

11. de Lauture, H., Cases, E., Dubost, P. et al. Concentrations of cholesterol, uric acid, urea, glucose and creatinine in a population of 50,000 active individuals. In Siest, G. (ed) Reference Values in Human Chemistry. Karger, Basle, 1973, p. 141-152. formulae to calculate a patient's glomerular filtration rate $/ 70 \mathrm{~kg}$ body weight each time a serum creatinine result is reported. The situations where these formulae may be invalid could be briefly outlined on the report.

Measurement of serum creatinine concentration is amongst the most commonly performed biochemical tests in hospital practice. It appears that much of the information about renal function that this test could provide has so far been unavailable to the majority of clinicians.

\section{Acknowledgement}

I am grateful to Mrs S. Taylor for her help with statistical analyses and with preparation of the illustration.

12. Wilding, P. Rollason, J.G. \& Robinson, D. Patterns of change of various biochemical constituents detected in a well population screening. Clin Chim Acta 1972, 41: 375-397.

13. Moniz, C.F., Nicolaides, K.H., Bamforth, F.J. et al. Normal reference ranges for biochemical substances relating to renal, hepatic and bone function in fetal and maternal plasma throughout pregnancy. $J$ Clin Pathol 1985, 38: 468-475.

14. Barratt, T.M. \& Round, J.M. Creatinine in plasma. In: Clayton, B.E., Jenkins, P., Round J.M. (eds) Paediatric Chemical Pathology: clinical tests and reference ranges. Blackwell Scientific Publications, Oxford, 1980, p. 60-63.

15. Sims, E.A.H. \& Krantz, K.E. Serial studies of renal function during pregnancy and in the puerperium in normal women. J Clin Invest 1958, 37: 1764-1774.

16. Hallynck, T., Soep, H.H., Thomis, J. et al. Prediction of creatinine clearance from serum creatinine concentration based on lean body mass. Clin Pharmacol Ther 1982, 30: 414-421.

17. Mawer, G.E., Knowles, B.R., Lucas, S.B., Stirland, B.M. \& Tooth, J.A. Computer assisted prescribing of kanomycin for patients with renal insufficiency. Lancet 1972, i: 12-15.

18. Cockcroft, D.W. \& Gault, M.H. Prediction of creatinine clearance from serum creatinine. Nephron 1976, 16: 31-41.

19. Beranek, P. \& Gabriel, R. Creatinine clearance without urine. Abstracts of the European Dialysis and Transplant Association 1985:62.

20. Jelliffe, R.W. Estimation of creatinine clearance when urine cannot be collected. Lancet 1971, i: 975-976.

21. Schwartz, G.J., Haycock, G.B., Edelmann, C.M. \& Spitzer, A. A simple estimate of glomerular filtration rate from plasma creatinine concentration in children. J Pediatr 1976, 58: 259-263. 
22. Siersback-Nielsen, K., Hansen, J.M., Kampmann, J. \& Kristensen, M. Rapid evaluation of creatinine clearance. Lancet 1971, i: 1133-1134.

23. Wheeler, L.A. \& Scheiner, L.B. Clinical estimation of creatinine clearance. Am J Clin Pathol 1979, 72: 2731.

24. Jelliffe, R.W. \& Jelliffe, S.M. A computer programme for estimating $\mathrm{Ccr}$ from unstable serum creatinine levels, age, sex and weight. Mathematical Biosci 1972, 14: 17-24.

25. Gabriel, R. Time to scrap creatinine clearance? $\mathrm{Br}$ Med J 1986, 293: 1119-1121.

26. Payne, R.B. \& Morgan, D.B. Sodium, water and acidbase balance: teaching transient and steady states. Med Educ 1977, 11: 133-135.

27. Jelliffe, R.W. \& Jelliffe, S.M. Estimation of creatinine clearance from changing serum creatinine levels. Lancet 1971, ii: 710.

28. Mayersohn, M., Conrad, K.A. \& Achari, R. The influence of a cooked meat meal on creatinine plasma concentration and creatinine clearance. $\mathrm{Br} J$ Clin Pharmacol 1983, 15: 227-230.

29. Statland, B.E., Winkel, P. \& Bokelund, H. Factors contributing to intra-individual variation of serum constituents: 1. Within day variations of serum constituents in healthy persons. Clin Chem 1973, 19: 1374-1379.

30. Jacobsen, F.K., Christensen, C.K., Mogensen, C.E. et al. Evaluation of kidney function after meals. Lancet 1980, i: 319 .
31. Statland, B.E. Winkel, P. \& Bokelund, H. Factors contributing to intra-individual variation of serum constituents: 2. Effect of exercise and diet on variations of serum constituents in healthy subjects. Clin Chem 1973, 19: 1380-1383.

32. Levene, P.A. \& Kristeller, L. Factors regulating the creatinine output in man. Am J Physiol 1909, 24; 4565.

33. Osberg, I.M. \& Hammond, K.B. A solution to the problem of bilirubin interference with the kinetic Jaffe method for serum creatinine. Clin Chem 1978, 24: 1169-1170.

34. Lebel, R.R., Gutmann, F.D., Mazumdar, D.C. et al. Creatinine determination in ketosis. $N$ Engl $\mathrm{J}$ Med 1984, 310: 1671.

35. Burry, H.C. \& Dieppe, P.A. Apparent reduction of endogenous creatinine clearance by salicylate treatment. $\mathrm{Br}$ Med J 1976, ii: 16-17.

36. Shouval, D., Ligumsky, M. \& Ben-Ishay, D. Effects of co-trimoxazole on normal creatinine clearance. Lancet 1978, i: 244-245.

37. Larsson, R., Bodemar, G., Kagedal, B. et al. The effects of cimetidine (Tagamet) on renal function in patients with renal failure. Acta Med Scand 1980, 208: 27-31. 